VLs Rates among patients ranged from 5000 to 10 million copies $/ \mathrm{ml}$, with a large majority of patients $(42.55 \%)$ whom VL was estimated from 10000 and 100000 copies $/ \mathrm{ml}$. Most prevalent HIV-1 subtypes were subtype B (74\%); and CRF02_G (26\%). Drug resistance exploration showed that $17 \%$ of the studied group carry at least one resistant mutation that confers resistance to non-nucleoside reverse transcriptase inhibitors (NNRTIs), and 13\% have at least one NRTI resistance mutation, while no major resistance mutation was detected for protease inhibitors (IPs). Detected mutations were as follows: M41L, K70E, M184V, L210W and T215C/D/S, responsible for nucleoside RT inhibitor (NRTI) resistance; K103N/S M230L and V106I, responsible for non-nucleoside RT inhibitor (NNRTI) resistance; M46L and L90M, responsible for protease inhibitor (PI) resistance.

The primary resistance rate observed in the study group was estimated at $8.5 \%(4 / 47)$. This rate describes the primary resistance level in this region as a moderate level (between 5 and $15 \%$ ), requiring continuous monitoring of resistance in patients immediately after diagnosis of infection and prior to initiation of treatment antiretroviral.

Disclosure of interest statement Nothing to declare.

\section{P17.29 UGT1A1*6 POLYMORPHISMS ARE PREDICTIVE OF HIGH PLASMA CONCENTRATIONS OF DOLUTEGRAVIR IN JAPANESE INDIVIDUALS}

${ }^{1} \mathrm{H}$ Yagura*, ${ }^{2} \mathrm{D}$ Watanabe, ${ }^{2} \mathrm{M}$ Ashida, ${ }^{1} \mathrm{H}$ Kushida, ${ }^{1} \mathrm{~K}$ Tomishima, ${ }^{2} \mathrm{~K}$ Hirota, ${ }^{2} \mathrm{M}$ Ikuma, ${ }^{2} \mathrm{~K}$ Yajima, ${ }^{2} \mathrm{D}$ Kasai, ${ }^{2} \mathrm{Y}$ Nishida, ${ }^{2} \mathrm{~T}$ Uehira, ${ }^{3} \mathrm{M}$ Yoshino, ${ }^{2} \mathrm{~T}$ Shirasaka. ${ }^{1}$ Department of Pharmacy, National Hospital Organization Osaka National Hospital; ${ }^{2}$ AIDS Medical Center, National Hospital Organization Osaka National Hospital; ${ }^{3}$ Department of Pharmacy, National Hospital Organization Himeji Medical Center

\subsection{6/sextrans-2015-052270.607}

Background Dolutegravir (DTG), an HIV integrase inhibitor, is metabolised mainly by glucuronidation via UDP-glucuronosyltransferase 1A1 (UGT1A1). Several UGT1A1 polymorphisms have been correlated with UGT1A1 expression level or enzymatic activity. We compared the effect of two polymorphic alleles in this gene, UGT1A1*6 and UGT1A1*28, on plasma DTG concentrations in Japanese HIV-1-infected patients.

Methods The plasma trough DTG concentration was measured in 69 HIV-1 patients taking DTG at Osaka National Hospital, and UGT1A1 genetic screening ( $* 6$ and $* 28)$ was performed. UGT1A1 was genotyped using the sequence method. Plasma was sampled immediately before taking DTG, and plasma DTG concentrations were determined using a liquid chromatography-mass spectrometry.

Results In the 69 patients who received DTG, the frequencies of UGT1A1*6 and UGT1A1*28 were $23 \%$ and $13 \%$, respectively. The plasma trough concentrations of DTG in patients homozygous for UGT1A1 $\%$ ( $\mathrm{n}=7$, median: $1.4 \mu \mathrm{g} / \mathrm{mL}$ ) were significantly higher those in the patients carrying the normal allele (n $=32$, median: $0.89 \mu \mathrm{g} / \mathrm{mL} ; \mathrm{p}=0.011$ ). The plasma trough concentrations of DTG in patients homozygous for UGT1A1*28 (n $=3$, median: $1.2 \mu \mathrm{g} / \mathrm{mL}$ ), compound heterozygous for UGT1A1*6 and UGT1A1*28 ( $\mathrm{n}=2,0.98$ and $1.2 \mu \mathrm{g} / \mathrm{mL}$, respectively), and heterozygous for UGT1A1*6 and UGT1A1*28 ( $\mathrm{n}=15$ and 10, median: 1.1 and $1.0 \mu \mathrm{g} / \mathrm{mL}$, respectively) were not significantly different from those in the patients homozygous for the normal allele.

Conclusion The plasma trough concentration of DTG was significantly higher in patients who were homozygous for UGT1A1*6 than in those with the normal allele. This finding suggests that the presence of UGT1A1*6 influences the plasma DTG concentration.

Disclosure of interest statement Authors do not have any commercial or other association that might pose a conflict of interest.

\section{P17.30 A FEASIBILITY STUDY ASSESSING FERTILITY IN HIV- SERODISCORDANT COUPLES ATTEMPTING PREGNANCY IN KISUMU, KENYA}

${ }^{1} \mathrm{O}$ Mmeje*, ${ }^{2} \mathrm{~A}$ Murage, ${ }^{3} \mathrm{~B}$ Njoroge, ${ }^{4} \mathrm{~S}$ van der Poel, ${ }^{5,6} \mathrm{CR}$ Cohen. ${ }^{1}$ University of Michigan, Department of Obstetrics and Gynecology; ${ }^{2}$ Aga Khan University, Nairobi, Kenya; ${ }^{3}$ Kenya Medical Research Institute (KEMRI), Centre for Microbiology Research; ${ }^{4}$ Department of Reproductive Health and Research Including the HRP Special Research Programme, World Health Organization, Geneva, Switzerland; '5Family AIDS Care and Education Services (FACES); ${ }^{6}$ University of California, San Francisco, Department of Obstetrics, Gynecology \& Reproductive Sciences

\subsection{6/sextrans-2015-052270.608}

Background HIV-serodiscordant couples desiring children face the difficult choice between risking transmission through condomless intercourse to become pregnant and the social stigmatisation of being childless. In sub-Saharan Africa, 14.3 million women are HIV-infected and many exercise their reproductive right to achieve pregnancy; however, though often overlooked, fertility problems due to tubal damage, menstrual cycle irregularities, and diminished ovarian reserve may affect these women.

Methods A study of female positive/male negative ( $++/ \hat{\delta}-)$ HIVserodiscordant couples using timed vaginal insemination (TVI) as a safer method of conception was conducted in Kenya. A fertility evaluation was offered to couples unable to achieve pregnancy after six cycles of TVI. Men completed a semen analysis and women a hystero-salpingo-contrast-sonography (HyCoSy) to investigate the uterus, ovaries, and fallopian tubes.

Results Fifteen of $23++/ \hat{\jmath}$ - HIV-serodiscordant couples were eligible for fertility evaluations; 14 consented; and seven completed male and female fertility assessments. The mean age of the men and women who completed fertility evaluations was 33.7 (std. dev. $=4.58$ ), and 30 (std. dev. $=5.41$ ) years, respectively. Fertility evaluations determined that three women had bilateral fallopian tube occlusion with one male partner having decreased sperm motility; and four women had unilateral/bilateral fallopian tube patency. Seven couples declined or were unable to complete a full couple evaluation. Of the 14 women evaluated, six $(46 \%)$ had an HIV RNA viral load ( $\geq 400$ copies/ $\mathrm{mL})$; six (46\%) had an undetectable HIV RNA viral load; and two declined serum assessment.

Conclusion Amongst HIV-serodiscordant couples attempting to achieve pregnancy, underlying male and/or female factor infertility and inadequate viral suppression may be present. As safer conception interventions are integrated into HIV care programs, a fertility evaluation should be offered, if feasible and available, to HIV-affected individuals/couples planning a pregnancy, to optimise their conception efforts, decrease HIV transmission, and assess their potential to conceive without assistance.

Disclosure of interest statement This research was supported in part by a grant from the National Institutes of Health (NIH), University of California San Francisco-Gladstone Institute of Virology and Immunology Centre for AIDS Research (P30 AI027763) and the United Nations jointly sponsored HRP Special Research Programme. O. Mmeje was partly supported by NIH/T32-5T32AI065388-03. 